\title{
On Relative Stability of Selected Hard Tetramer Solids
}

\author{
M. Kowalik, K.V. Tretiakov, K.W. Wojciechowski \\ Institute of Molecular Physics, Polish Academy of Sciences \\ ul. Smoluchowskiego M. 17, PL-60-179 Poznań, Poland \\ e-mails: \{kowalik/kvt/kww\}@ifmpan.poznan.pl; kww@man.poznan.pl
}

(Received: 29 October 2010; revised: 10 December 2010; accepted: 13 December 2010; published online: 17 December 2010)

\begin{abstract}
The Einstein crystal method was used to determine free energy differences between some crystalline structures of hard, homonuclear tetramers. The tetramers, each built of four identical hard spheres centered on vertices of a regular tetrahedron of sides equal to the sphere diameter, were arranged in such a way that the spheres formed the fcc lattice at close packing. Various sample sizes were studied and the results were extrapolated to the thermodynamic limit. It was found that the simplest structure of the tetramers, a simple cubic lattice of molecular mass centres with all the molecules having the same orientation, shows the highest free energy amongst the studied ones. The most stable structure of the studied ones was also found.
\end{abstract}

Key words: Monte Carlo method, hard multispheres, free energy, Einstein crystal method, molecular crystals

\section{INTRODUCTION}

More than 50 years of studies of systems which particles interact via hard-body potential (infinite when some particles overlap and zero otherwise) proved that many complex physical phenomena can have purely geometrical origin. Pioneering works on hard sphere systems $[1,2]$ provided a deeper insight into freezing of rare gases $[3,4]$. Other papers showed that even very crude molecular models such as hard spherocylinders [5, 6], ellipsoids [7-9], and dumbbells [10-13] exhibit surprisingly rich phase diagrams and they are able to mimic many phases encountered in real liquid crystals and solids.

From their very nature hard-body systems serve as a convenient reference systems in which short range repulsion interactions and thus packing effects play dominant role, such as fluids. They were also successfully used for studying phase behaviour of propane [14] and benzene [15, 16]. Also they proved useful in investigating phase equilibria of chiral molecules and enantiomeric mixtures $[17,18]$.

One of the most significant characteristics of a system is its phase behaviour. To study phase diagrams of physical systems, the knowledge of the Helmholtz free energy [19] at given conditions is necessary, since only the system with minimal free energy will be thermodynamically stable. In general, the analytical determination of the free energy is difficult and often impossible, even for simple theoretical models. Fortunately, computer simulations provide a useful tool for determining phase behaviour of model systems. In particular, they were applied to solve the long standing problem of thermodynamic stability of the hard sphere system. It is well known that hard spheres can be arranged into infinitely many various close packed structures. The most noticeable are two of them: (a) the hexagonal close packed (hcp) and (b) the face centered cubic (fcc) structure. It was found that the free energy of fcc structure is lower then the free energy of hcp structure [20-23]. Moreover, the fcc lattice is the most stable one of all close packed structures of hard spheres [24].

In the present paper, we consider a few structures formed by hard tetramers of tetraedric symmetry. The hard tetramer consists of four adjacent hard spheres (each of diameter $\sigma$ which is further considered as a unit of length) which centers are located in the vertices of regular tetrahedron. The simplest structure of such molecules is that in which the mass centres of the tetramers are arranged into a simple cubic lattice and the molecules are identically oriented, further referred to as the $\mathrm{FCC1}$, in the way in which their 'atoms' (spheres) form the fcc lattice. Some alterations are made to it (described later in the detail) to obtain new structures, denoted by $\mathrm{FCC} i, i=2, \ldots, 6$. However, in all investigated structures the 'atoms' remain arranged into the fcc lattice, at the close packing.

We chose such a model molecule and its structures for the following reasons. As it was mentioned above, the fcc structure has the lowest free energy for hard spheres. It is 
then not unreasonable to assume that the same will be true also for multisphere bodies which can be packed in the same way as hard spheres. In contrast to hard dimers [25-27] and hard trimers [28] which crystallize into degenerate crystalline phases, hard tetramers cannot be easily (if at all) arranged into analogous structure. Hence, the degeneracy per particle (if non-zero) cannot be large and it is meaningful to assume that their 'ground state' corresponds to a periodic crystal what significantly simplifies free energy calculations. We start with a highly symmetric structure what reduces the number of parameters required for description of their structural and (probably also) thermodynamic properties.

The aim of the studies described in this paper is to determine how modifications of a simple, highly symmetric structure affect the value of its free energy. One of consequences of these studies is determination of the most stable structure (i.e., the one of the lowest free energy) amongst the studied ones. This research is a first step in solving a more general problem of determination of the stable solid phase of hard tetramers. The answer to the last question should help in understanding stability of some molecular phases.

\section{METHOD DESCRIPTION}

A standard technique of determining the free energy of model solids is the so-called Einstein-crystal method [29, 30]. Not only is it simple but it is quite precise [23], as well. Although initially used for spherical molecules, it can be also extended to non-spherical molecules in a quite straightforward manner [8, 14, 15, 18, 31-33]. The basic idea standing behind this method is to find a reversible path linking the solid under consideration with the noninteracting Einstein crystal of the same structure, the free energy of which is known exactly. For a system consisted of $N$ nonlinear molecules (such as tetramers) appropriate linkage between the solid under consideration and the reference state can be achieved by replacing the original hard-body potential $U_{0}$ with the following [33] one:

$$
\begin{aligned}
U=U_{0} & +U_{\mathrm{E}, \mathrm{id}}=U_{0}+\lambda_{1} \sum_{i=1}^{N}\left(\mathbf{r}_{i}-\mathbf{r}_{i}^{0}\right)^{2}+ \\
& +\lambda_{2} \sum_{i=1}^{N}\left[\sin ^{2} \theta_{i}+\sin ^{2} \psi_{i}\right],
\end{aligned}
$$

where $\mathbf{r}_{i}-\mathbf{r}_{i}^{0}$ is displacement of molecule $i$ from its equilibrium position $\mathbf{r}_{i}^{0}, \theta_{i}$ is the angle between body-fixed $z$-axis of the $i$-th particle and its average direction. Analo- gously, $\psi_{i}$ is the angle formed between body-fixed $x$-axis of the particle $i$ and its average direction. Parameters $\lambda_{i}$ $(i=1,2)$ measure the strength of harmonic coupling of molecules to their equilibrium positions and orientations, respectively. It is usual practice to set their numerical values to be equal, thus both of them will be hereinafter referred to as $\lambda$.

For numerical reasons [29, 30], the free energy calculations are carried out with fixed center of mass of the system. In such a case, the Helmholtz free energy of the solid can be expressed as $[34,35]$

$$
f=f_{\mathrm{E}, \mathrm{id}}^{\mathrm{CM}}+\Delta f_{\mathrm{E}}^{\mathrm{CM}}+\Delta f_{\lambda}^{\mathrm{CM}}+\Delta f
$$

where $f \equiv \beta f / N$ and $\beta=1 / k_{\mathrm{B}} T$ with $T$ being the temperature. Symbol $f_{\mathrm{E}, \mathrm{id}}^{\mathrm{CM}}$ denotes the free energy of the ideal (non-interacting) Einstein crystal with fixed center of mass. For atomic solids it can be computed analytically [36]. In case of molecular solids numerical estimation are usually required [37] or for large values of $\lambda$ some approximations can be made $[14,38]$. The second term $\Delta f_{\mathrm{E}}^{\mathrm{CM}} \equiv f_{\mathrm{E}, \mathrm{sol}}^{\mathrm{CM}}-f_{\mathrm{E}, \mathrm{id}}^{\mathrm{CM}}$ represents the free energy difference between the interacting and ideal Einstein crystal, both with the center of mass fixed. For fixed values of coupling parameters it can be written as

$$
\Delta f_{\mathrm{E}}^{\mathrm{CM}}=-\frac{1}{N} \ln \left\langle\exp \left(-\beta U_{0}\right)\right\rangle_{\mathrm{E}, \mathrm{id}} .
$$

The average is taken over the states of the noninteracting Einstein crystal. For sufficiently large values of $\lambda$ this contribution is expected to be very small, practically negligible.

The next term in Eq. (2), $\Delta f_{\lambda}^{\mathrm{CM}} \equiv f_{\mathrm{sol}}^{\mathrm{CM}}-f_{\mathrm{E} \text {,sol }}^{\mathrm{CM}}$, represents the free energy change related to switching off the harmonic coupling, i.e. the free energy difference between solid under consideration and the interacting Einstein crystal (both with center of mass fixed). It can be written as [35]

$$
\begin{gathered}
\Delta f_{\lambda}^{\mathrm{CM}}=-\frac{\beta}{N} \int_{0}^{\lambda} d \lambda^{\prime}\left\langle\frac{\partial U}{\partial \lambda^{\prime}}\right\rangle_{N V T}= \\
=-\frac{\beta}{N} \int_{0}^{\lambda} d \lambda^{\prime}\left\langle\left.\frac{1}{\lambda^{\prime}} U_{\mathrm{E}, \mathrm{id}}\right|_{\lambda_{1}=\lambda_{2}=\lambda^{\prime}}\right\rangle_{N V T} .
\end{gathered}
$$

Symbol $\langle\ldots\rangle_{N V T}$ denotes averages taken over the canonical ensemble.

Finally, the last term $\Delta f \equiv f_{\text {sol }}-f_{\text {sol }}^{\mathrm{CM}}$ represents free energy change between an unconstrained solid and the solid with fixed center of mass. Its detailed derivation can be found in Refs. [30, 35, 36, 39]. 


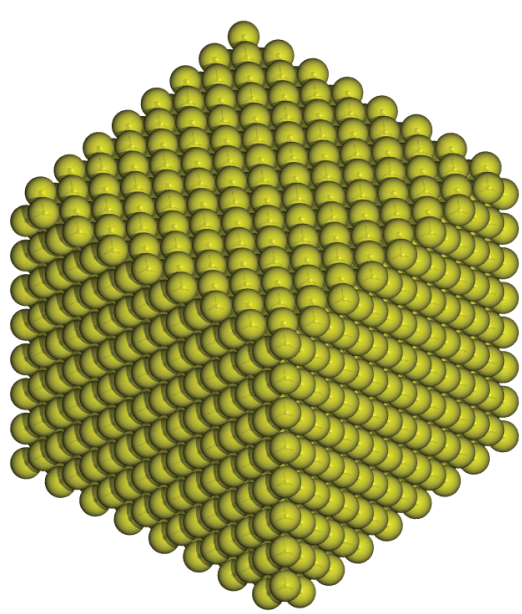

(a) FCC1

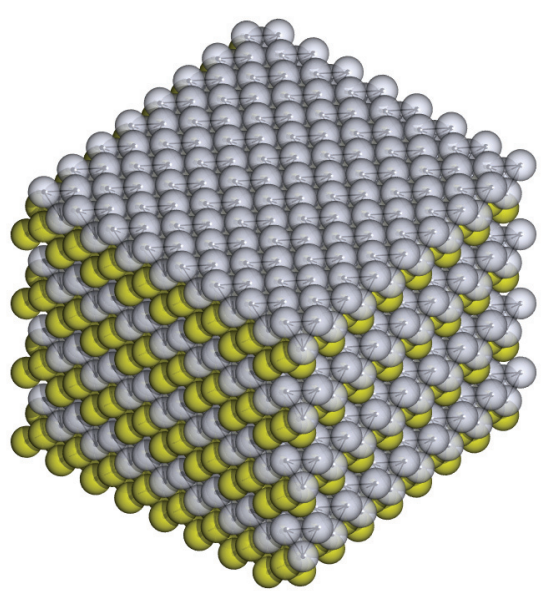

(c) FCC3

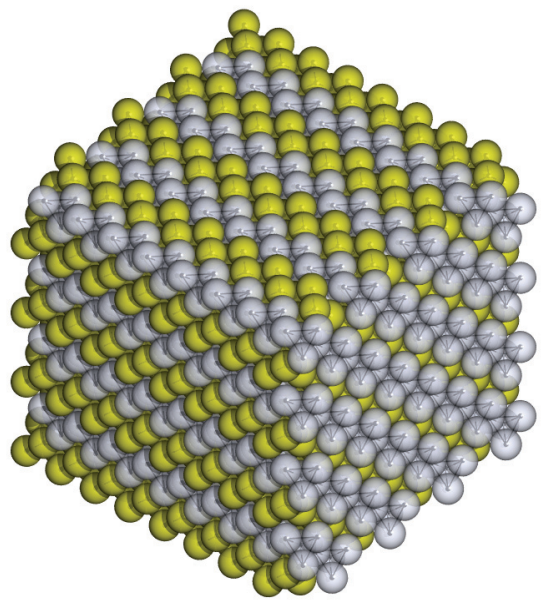

(e) FCC5

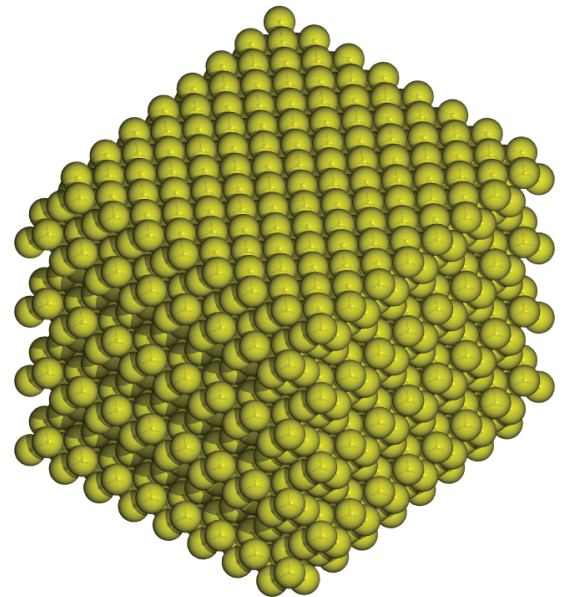

(b) $\mathrm{FCC} 2$

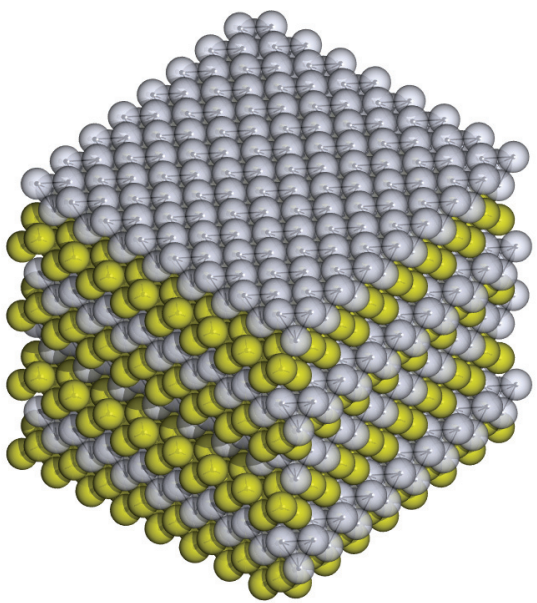

(d) FCC4

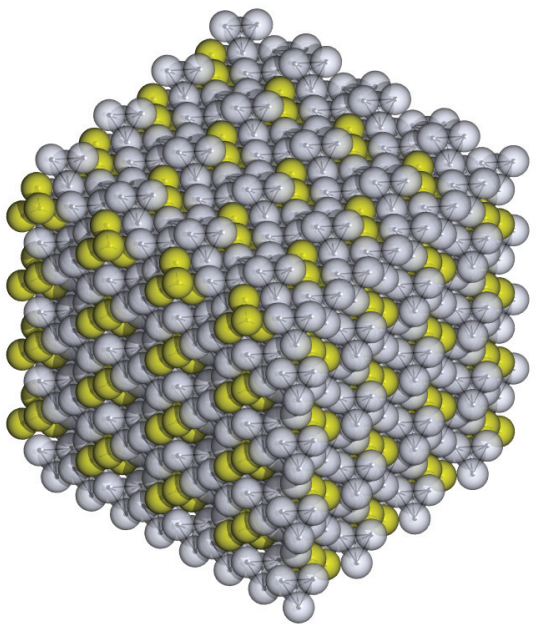

(f) FCC6

Fig. 1. Closed packed crystalline structures of hard tetramers which 'atoms' form the fcc lattice. The tetramers with unchanged orientations (relative to FCC1) are yellow. The tetramers rotated about $\pi / 2$ around one of their 2 -fold axes are grey 


\section{RESULTS AND DISCUSSION}

We considered six different crystalline hard tetramer structures in which 'atoms' of the molecules constitute fcc lattice at close packing limit. They are illustrated of Fig. 1. In FCC1 structure tetramers are arranged in simple cubic lattice with the lattice constant $a=\sqrt{2}$ and their 2-fold axes are parallel to $z$-axis. It is worth noticing that all subsequent structures, counting from FCC2 to FCC6, are modifications of this structure. In FCC2 every even $x y$-plane of tetramers was shifted by the vector $[a / 2, a / 2,0]$; in FCC3 every even $x y$-plane of tetramers was shifted by $[a / 2,0,0]$ and the tetramers in those planes were at the same time rotated by $\pi / 2$ around any of their 2 -fold axes. Analogously, in FCC4 structure, in every second plane tetramers were shifted alternately by vectors $[a / 2,0,0]$ and $[0, a / 2,0]$ respectively relative to $\mathrm{FCC} 1$ and rotated as described above.

In the case of FCC5 and FCC6 structures rows of tetramers were shifted instead of their planes and the molecules were rotated in the shifted rows.

In FCC5, in every odd plane every even row of tetramers was shifted by the vector $[a / 2,0,0]$ and tetramers were rotated as before. In every even plane the opposite has been done - every odd row was shifted by the same vector and tetramers were rotated appropriately.

In the last structure (FCC6): (a) tetramers in every odd row in every odd $x y$-plane were shifted by the vector $[a / 2$, $0,0]$; (b) tetramers in every even row in every even $x y$-plane were shifted by the vector $[0, a / 2,0]$; (c) tetramers in every even row in every odd $y z$-plane were shifted by the vector $[0,0, a / 2]$. As before, tetramers in every shifted row were rotated by $\pi / 2$ around any of their 2 -fold axes.

At the initial stage of free energy calculations, all investigated structures were equilibrated in a variant of constant pressure $\mathrm{MC}$ simulations in which simulation box could vary both in size and shape. Every $N p T$ run consisted of $1.1 \times 10^{7} \mathrm{MC}$ cycles. The acceptance ratios were kept at level of $30 \%$ and $20 \%$ for translational-rotational moves and the volume changes, respectively. The dimensionless pressure $p^{*}=p \sigma^{3} / k_{\mathrm{B}} T$ was set to 6.4752 to ensure that the final reduced density $\rho^{*}=\rho / \rho_{0}$ (where $\rho_{0}$ is the density of the structure at close packing) will be close to 0.7500 - value which lies fairly close to the density at which hard tetramer system melts spontaneously. Those runs allowed us to determine precisely the equilibrium structures for free energy calculations at the given density by averaging the box parameters and both positions and orientations of the particles.

After obtaining reliable reference structures and following the procedure described in [30], a 20-point Gauss-Le- gendre (GL) quadrature was used to estimate the integral in Eq. (4). For each structure, simulation in $N V T$ ensemble consisted of $120000 \mathrm{MC}$ cycles (20 000 for equilibration and 100000 for averaging) for each GL point. The acceptance ratio for translational-orientational moves was kept at the level of $30 \%$. The parameter $\lambda$ was equal 32000 .

To estimate the $N$-dependence of the free energy term, $\Delta f_{\lambda}^{\mathrm{CM}}$, samples consisting of $N=64,216,512$ i 1000 molecules were used for each structure. The obtained results are illustrated in Fig. 2.

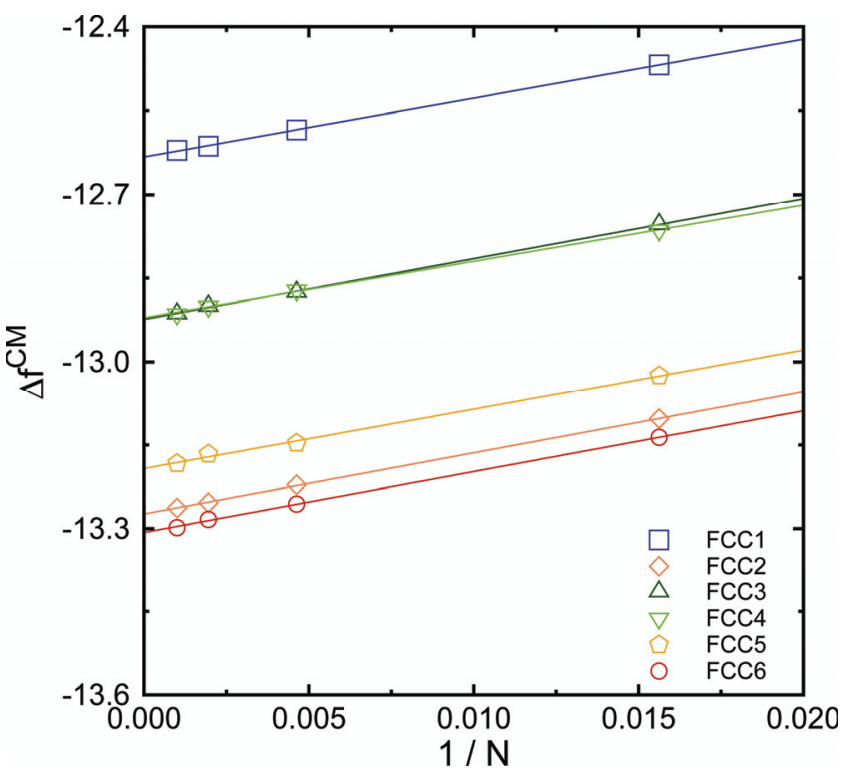

Fig. 2. $N$-dependence of the free energy term, $\Delta f_{\lambda}^{\mathrm{CM}}$, for the hard tetramer crystalline structures obtained at $\rho=0.7500$ with $\lambda=32000$ using 20-point GL quadrature to evaluate the integral in Eq. (4). The lines present linear fits to the data depicted

Since we are interested only in relative differences between the free energies of the investigated structures we can limit our considerations only to the comparison of the values of $\Delta f_{\lambda}^{\mathrm{CM}}$ expressed in Eq. (4). The value of $\lambda$ was chosen to make the term $\Delta f_{\mathrm{E}}^{\mathrm{CM}}$ negligible and the rest of correction terms are identical in case of all structures.

As one can see, the FCC1 structure, exhibiting the highest symmetry of the studied structures of the tetramers, has the highest free energy. Shifting the $x y$-planes of tetramers alone along $x$-axis (FCC3) or alternately along $x$ - and $y$-axes (FCC4) lowers the free energy significantly. Moreover, shifting the $x y$-planes of tetramers along direction [110] of the fcc lattice (FCC2) lowers free energy even more. Shifting only the rows also lowers the free energy in significant way. In fact, structure FCC6 has the lowest free energy among the investigated structures. 


\section{SUMMARY}

The free energy of the simple cubic structure of hard tetramers which 'atoms' (hard spheres) are arranged into the fcc lattice (with lattice constant $a=\sqrt{2}$ at close packing limit) was compared to the free energies of five other crystalline structures of hard tetramers. The latter structures were obtained by simple modifications of the initial structure like moving the planes or rows of the tetramers.

The simulations indicate that moving only planes as well as shifting the rows of tetramers leads to significant decrease of free energy value. The latter method was used to design the structure FCC6 (see Fig. 1f) which appeared to have the lowest value of free energy of all. However there are infinitely many various periodic decorations of the different close packed structures of hard spheres. Thus, although the FCC6 is the most stable of the studied structures, we cannot state definitely that this structure is thermodynamically stable.

The free energy value of the FCC6 structure should be at least compared with a few simple structures of hard tetramers which atoms constitute the hep lattice. This issue will be a subject of future research.

\section{Acknowledgements}

Part of this work was supported by the Polish Ministry of Science and Education with the grants N202 070 32/1512 and NN202 261438. Some simulations were performed at the Poznań Supercomputing and Networking Center (PCSS).

\section{References}

[1] B.J. Alder, T.E. Wainwright, J. Chem. Phys. 27, 1208 (1957).

[2] W.W. Wood, J.D. Jacobson, J. Chem. Phys. 27, 1207 (1957).

[3] H.C. Longuet-Higgins, B. Widom, Mol. Phys. 8, 549 (1964).

[4] A.D.J. Haymet, Science 236, 1076 (1987).

[5] J.A.C. Veerman, D. Frenkel, Phys. Rev. A 41, 3237 (1990).

[6] R. Blaak, D. Frenkel, B.M. Mulder, J. Chem. Phys. 110, 11652 (1999).
[7] D. Frenkel, B.M. Mulder, J.P. McTague, Phys. Rev. Lett. 52, 287 (1984).

[8] D. Frenkel, B.M. Mulder, Mol. Phys. 55, 1171 (1985).

[9] B.M. Mulder, D. Frenkel, Mol. Phys. 55, 1193 (1985).

[10] S.J. Singer, R. Mumaugh, J. Chem. Phys. 93, 1278 (1990).

[11] C. Vega, E.P.A. Paras, P.A. Monson, J. Chem. Phys. 96, 9060 (1992).

[12] C. Vega, E.P.A. Paras, P.A. Monson, J. Chem. Phys. 97, 8543 (1992).

[13] C. Vega, P.A. Monson, J. Chem. Phys. 107, 2696 (1997)

[14] W.N. Shen, P.A. Monson, J. Chem. Phys. 103, 9756 (1995).

[15] J.W. Schroer, P.A. Monson, J. Chem. Phys. 112, 8950 (2000).

[16] J.W. Schroer, P.A. Monson, J. Chem. Phys. 114, 4124 (2001).

[17] J. Largo, C. Vega, L.G. MacDowell, J.R. Solana, Mol. Phys. 100, 2397 (2002).

[18] M. Cao, P.A. Monson, J. Chem. Phys. 122, 05405 (2005).

[19] K. Huang, Statistical Mechanics (John Wiley \& Sons, New York, 1963).

[20] L.V. Woodcock, Nature 385, 141 (1997).

[21] L.V. Woodcock, Nature 388, 236 (1997).

[22] R.J. Speedy, J. Phys.: Condens. Matter 10, 4387 (1998).

[23] K.V. Tretiakov, K.W. Wojciechowski, Phys. Rev. E 60, 7626 (1999).

[24] S.C. Mau, D.A. Huse, Phys. Rev. E 59, 4396 (1999).

[25] K.W. Wojciechowski, Mod. Phys. Lett. B 5, 1843 (1991).

[26] K.W. Wojciechowski, D. Frenkel, A.C. Brańka, Phys. Rev. Lett. 67, 3168 (1991).

[27] K.V. Tretiakov, K.W. Wojciechowski, J. Non-Cryst. Sol. 352, 4221 (2006).

[28] M. Kowalik, K.W. Wojciechowski, Phys. Stat. Solidi (b) 242, 626 (2005).

[29] D. Frenkel, A.J. Ladd, J. Chem. Phys. 81, 3188 (1984).

[30] D. Frenkel, B. Smit, Understanding molecular simulation (Academic Press, San Diego, 2002).

[31] E.G. Noya, C. Vega, J.P.K. Doye, A.A. Louis, J. Chem. Phys. 127, 054501 (2007).

[32] E.G. Noya, M.M. Conde, C. Vega, J. Chem. Phys. 129, 104704 (2008).

[33] E.G. Noya, C. Vega, J.P.K. Doye, A.A. Louis, J. Chem. Phys. 132, 234511 (2010).

[34] E. de Miguel, R.G. Marguta, E.M. del Río, J. Chem. Phys. 127, 154512 (2007).

[35] C. Vega, E. Sanz, J.L.F. Abascal, E.G. Noya, J. Phys.: Condens. Matter 20, 153101 (2008).

[36] J.M. Polson, E. Trizac, S. Pronk, D. Frenkel, J. Chem. Phys. 112, 5339 (2000).

[37] J. Chang, A.M. Lenhoff, S.I. Sandler, J. Chem. Phys. 120, 3003 (2004).

[38] C. Vega, P.A. Monson, J. Chem. Phys. 109, 9938 (1998).

[39] G. Navascués, E. Velasco, J. Chem. Phys. 132, 134106 (2010).

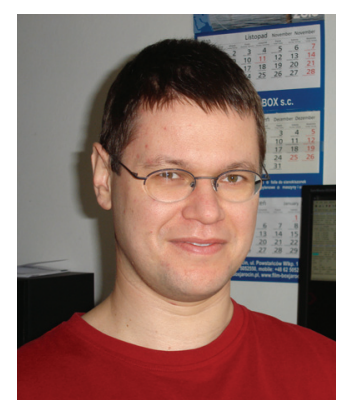

MikolaJ Kowalik, graduated in Theoretical Physics in 1999 from Adam Mickiewicz University in Poznań. Received his PhD degree in Physics in 2005 from Institute of Molecular Physics in Poznań. He specializes in computer simulations, focusing his interest on elastic properties of model systems interacting via hard-body potential. 


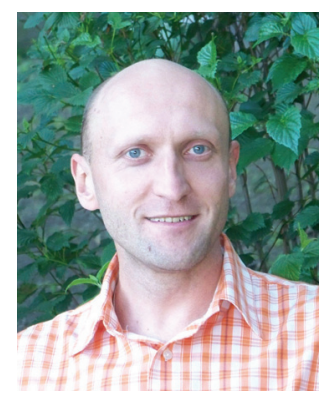

Konstantin V. Tretiakov graduated summa cum laude in Industrial Electronics from the The Pavel Sukhoi State Technical University (Gomel, Belarus) in 1994. He received his Ph.D. degree in Computational Physics from the Institute of Molecular Physics Polish Academy of Science (Poznan, Poland) in 2000. In 2002-2004, Dr. Tretiakov was a Postdoctoral Fellow (with Prof. S. Scandolo) in the Condensed Matter and Statististical Physics Section at the Abdus Salam International Center for Theoretical Physics (Trieste, Italy). In 2007-2009, Dr. Tretiakov worked with Prof. B. Grzybowski in the Department of Chemical and Biological Engineering at Northwestern University (Evanston IL, USA). He is a staff member of Prof. K. W. Wojciechowski group. Dr. Tretiakov specializes in statistical physics and computer simulation of many body systems, with research focusing on non-equilibrium self-assembling systems, transport properties of soft-matter, elastic properties of solids in particular materials exhibiting negative Poisson's ratio.

Krzysztof W. Wojciechowski is the Full Professor and the Head of the Nonlinear Dynamics and Computer Simulation Division at the Institute of Molecular Physics of the Polish Academy of Sciences. He received his MSc degree in physics and MSc degree in mathematics from the Adam Mickiewicz University in Poznań. He earned the PhD in physics from the Institute of Molecular Physics, where he also habilitated. His research interests concern, amongst other topics, statistical-mechanical properties of hard-body systems (statistical-mechanical geometry), algorithms for simulations of many-body systems, studies of various mechanisms leading to auxetic behaviour (negative Poisson's ratio) of condensed matter systems, applications of fractional derivative in physics, and exotic liquid crystalline phases. 